\title{
Application of Gaussia luciferase in bicistronic and non-conventional secretion reporter constructs
}

\author{
Christin Luft ${ }^{1}$, Jamie Freeman ${ }^{1}$, David Elliott ${ }^{1}$, Nadia Al-Tamimi ${ }^{1}$, Janos Kriston-Vizi ${ }^{1}$, Jacob Heintze ${ }^{1}$, \\ Ida Lindenschmidt ${ }^{1}$, Brian Seed ${ }^{2}$ and Robin Ketteler ${ }^{1 *}$
}

\begin{abstract}
Background: Secreted luciferases are highly useful bioluminescent reporters for cell-based assays and drug discovery. A variety of secreted luciferases from marine organisms have been described that harbor an $\mathrm{N}$-terminal signal peptide for release along the classical secretory pathway. Here, we have characterized the secretion of Gaussia luciferase in more detail.

Results: We describe three basic mechanisms by which GLUC can be released from cells: first, classical secretion by virtue of the N-terminal signal peptide; second, internal signal peptide-mediated secretion and third, non-conventional secretion in the absence of an N-terminal signal peptide. Non-conventional release of dNGLUC is not stress-induced, does not require autophagy and can be enhanced by growth factor stimulation. Furthermore, we have identified the golgi-associated, gamma adaptin ear containing, ARF binding protein 1 (GGA1) as a suppressor of release of dNGLUC.

Conclusions: Due to its secretion via multiple secretion pathways GLUC can find multiple applications as a research tool to study classical and non-conventional secretion. As GLUC can also be released from a reporter construct by internal signal peptide-mediated secretion it can be incorporated in a novel bicistronic secretion system.
\end{abstract}

Keywords: Non-conventional secretion, Gaussia luciferase, GGA1, Bicistronic expression, Signal peptide

\section{Background}

Secreted luciferases are versatile tools to investigate cell-based processes based on their high sensitivity, lack of toxicity, small size, wide dynamic range for quantitation and potential for multiplexing. The first secreted luciferase identified was isolated from the marine ostracod crustacean, Cypridina hilgendorfii, now named Vargula hilgendorfii [1]. Later, secreted luciferases were identified in the marine copepod crustaceans Gaussia princeps [2], Metridia longa [3], Metridia pacifica [4] and, more recently, from copepods of the Heterorhabdidae, Lucicutiidae and Augaptiloidae families [5,6]. With the exception of Vargula luciferase, that uses luciferin, all secreted luciferases utilize the substrate coelenterazine to convert light into a bioluminescent signal. Recently, a small, secreted luciferase termed

\footnotetext{
* Correspondence: r.ketteler@ucl.ac.uk

'Medical Research Council, Laboratory for Moleclar and Cell Biology, University College London, Gower Street, London WC1E 6BT, UK Full list of author information is available at the end of the article
}

Nanoluc engineered from a deep-sea shrimp luciferase has been developed that utilizes furimazine as substrate in an ATP-independent reaction [7].

One hallmark of secreted luciferases is their flash-type kinetics which has limited the use in drug discovery high-throughput screening applications. This hurdle has been overcome by engineering of Gaussia luciferase with glow-type characteristics [8-10]. Gaussia luciferase has been used to measure various cellular processes in cellbased assays as well as in vivo [11]. A split-GLUC system has been developed [12,13] and used for imaging of receptor-ligand interactions [14], virus-host protein interactions [15] and oligomer formation of amyloid beta peptide [16]. A highly useful property of Gaussia luciferase is its stability in body fluids such as blood and urine. This has facilitated the development of in vivo sensors for analysis of tumour growth [17] and caspase activation [18], among others. Secretion of Gaussia luciferase is mediated by a conventional $\mathrm{N}$-terminal signal peptide. 
Inducible release of Gaussia luciferase from an intracellularly retained version is possible [19], facilitating the development of intracellular biosensors. Two modifications are necessary for the development of such "luciferase release assays": one is the deletion of the N-terminal signal peptide (dNGLUC) and the second is the attachment of an anchor to dNGLUC, either by linkage to the actin cytoskeleton or other intracellular retained proteins $[18,19]$. The first luciferase release assay was used to monitor caspase 8 and 9 activation [19] and autophagy protease ATG4B activation [20]. Later, biosensors for caspase 3 [21], HCV protease [22] and caspase 1 [18] have been developed, all based on the proteolytic cleavage of a peptide sequence that is inserted between Gaussia luciferase and the anchor molecule, thus resulting in liberation and export of the luciferase from cells. The non-invasive nature of the luciferase release assay makes it a good assay for high-throughput screening and drug discovery applications and has been successfully used to identify molecular regulators of apoptosis and autophagy [20,23-26]. However the mechanism of the non-conventional secretion that underlies the luciferase release assay is poorly understood. In this study, we have identified molecular determinants that regulate non-conventional secretion of dNGLUC and have studied the role of autophagy in this process.

\section{Results}

\section{Non-conventional release of Gaussia luciferase}

This study examines the suitability of wild-type GLUC and dNGLUC, a deletion mutant without N-terminal signal peptide, as reporter systems to study protein secretion. Multiple tagged versions of GLUC and dNGLUC were generated as shown in Figure 1A. These include green fluorescent protein (GFP)-tagged versions of GLUC and dNGLUC as well as $\beta$ - actin tagged versions. First, it was confirmed that dNGLUC is efficiently released from cells in the absence of a signal peptide, whereas $\beta$-actin-tagged $\mathrm{dNGLUC}$ is retained inside cells (Figure 1B). One possible explanation is that dNGLUC harbors a new N-terminal secretion signal that would allow export from the cells. In order to test this hypothesis, dNGLUC was tagged with GFP to generate GFP-dNGLUC. GFP-dNGLUC was released from cells to a similar extent as dNGLUC and release for both dNGLUC and GFP-dNGLUC was sensitive to treatment with Brefeldin A, thus suggesting that ERGolgi trafficking is required (Figure 1B,C).

GFP- and $\beta$-actin-tagged versions of wild-type GLUC with an internal signal peptide between the two open reading frames as well as wt GLUC were also tested for release of luciferase activity from cells. As expected, wild-type GLUC was released at high levels from cells. In addition, GFP-tagged GLUC and $\beta$-actin-tagged GLUC were also secreted at moderate levels from cells
(Figure 1D), despite the absence of an $\mathrm{N}$-terminal signal peptide. Secretion of GFP-sp GLUC and $\beta$-actin- ${ }_{\text {SP }}$ GLUC was sensitive to treatment with Brefeldin A (Figure 1E). Unlike $\beta$-actin-dNGLUC without internal signal peptide, $\beta$-actin-sp GLUC with internal signal peptide was not retained in cells. One hypothesis is that an internal signal peptide is sufficient to mediate release of the downstream peptide sequence.

\section{Bicistronic reporter gene expression}

It has been shown before that internal signal peptides have the potential to result in secretion of the downstream gene and can be cleaved by signal peptidase [27]. To examine whether the internal signal peptide in GFP-

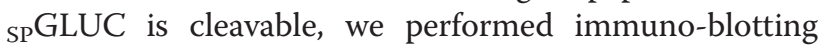
using a polyclonal antibody raised against the C-terminal part of GLUC. In untreated $293 \mathrm{~T}$ cells transfected with GLUC, we observed an expected band at $\sim 20 \mathrm{kDa}$ that increased in intensity upon treatment with Brefeldin A, consistent with accumulation of GLUC inside cells upon blockage of release (Figure 2A). For GFP-sPGLUC and $\beta$-actin-sPGLUC multiple bands were observed including a band at $47 \mathrm{kDa}$ and $62 \mathrm{kDa}$, respectively, that corresponds to the length of the full-length protein, as well as a band at $20 \mathrm{kDa}$ that corresponds in size to wild-type GLUC. Upon treatment with Brefeldin A, the $20 \mathrm{kDa}$ band increased in intensity, while the bands corresponding to full-length GFP-spGLUC or $\beta$-actin-spGLUC did not change in intensity. Therefore, we proposed that GFP-sPGLUC and $\beta$-actin-spGLUC are cleaved at the site of the signal peptide and the cleaved GLUC but not the full-length construct is released from cells. To test this hypothesis, we enriched supernatants from serum-free media and performed immuno-blotting of cell lysates and enriched supernatants in the presence or absence of Brefeldin A (Figure 2B). From cells transfected with GLUC, a strong band at $\sim 20 \mathrm{kDa}$ was observed in supernatants that was reduced upon treatment with Brefeldin A. Similarly, from cells transfected with GFP-spGLUC, a single band at $\sim 20 \mathrm{kDa}$ was observed that was significantly reduced in supernatants and increased in cell lysates upon treatment with Brefeldin A (Figure 2B). No band corresponding to full length GFP-spGLUC was observed in the supernatant, indicating the protein needs to be cleaved for secretion. We therefore conclude that the strong GFP-

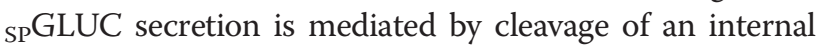
signal peptide.

\section{GLUC is heat-stable}

We noted that GLUC activity in supernatants was very stable and that the supernatants could be stored at room temperature for several days without apparent loss of luciferase activity. GLUC and dNGLUC activities were 
A

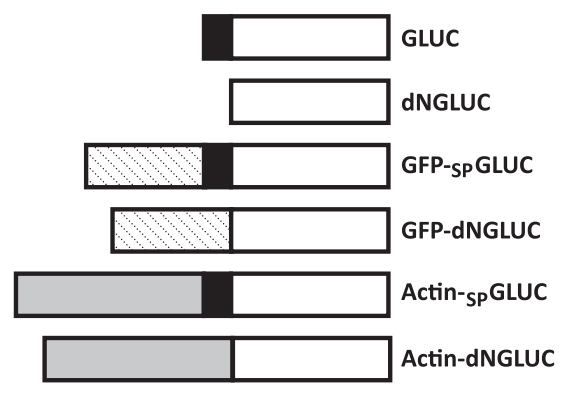

B

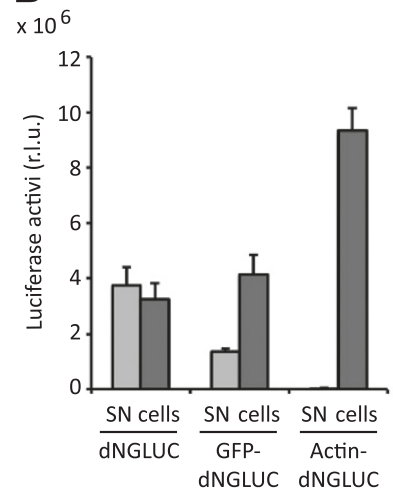

C

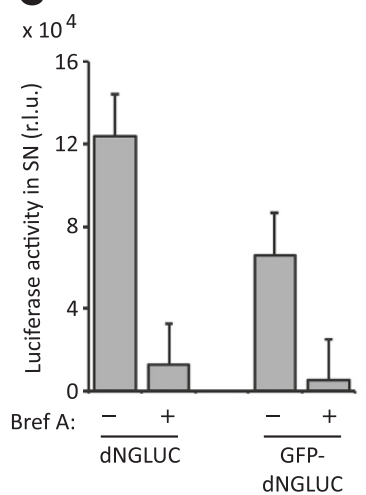

D

$\times 10^{7}$

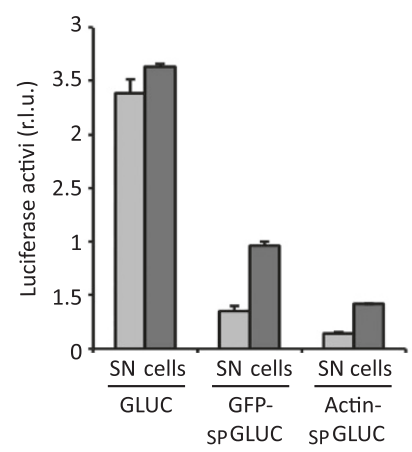

E

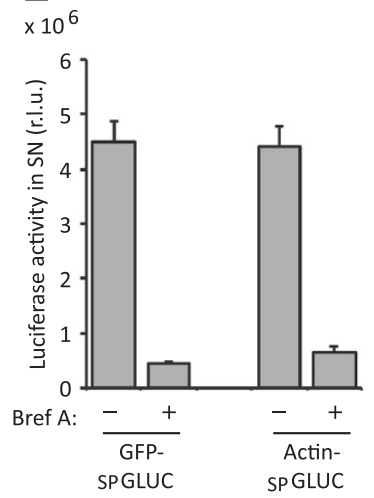

Figure 1 Three types of secretion for Gaussia luciferase activity. A, Constructs used in this study are wild-type Gaussia luciferase (GLUC), an $\mathrm{N}$-terminal deletion mutant that has no signal peptide (dNGLUC), GFP-tagged GLUC with an internal signal peptide (GFP-spGLUC), GFP-tagged dNGLUC and $\beta$-actin-tagged GLUC with and without an internal signal peptide (Actin-spGLUC, Actin-dNGLUC). The signal peptide is shown as a black box, GLUC without signal peptide is shown in white, GFP as a striped box and $\beta$-actin as a grey box. B, 293 T cells were transfected with dNGLUC, GFP-dNGLUC and $\beta$-actin-dNGLUC, supernatants and cell lysates were collected after $24 \mathrm{~h}$ and analyzed for luciferase activity. GLUC activity was measured as described in Methods. C, 293 T cells were transfected with dNGLUC and GFP-dNGLUC and cultured for $24 \mathrm{~h}$ prior to a media change. Fresh medium containing DMSO or $10 \mu \mathrm{g} / \mathrm{mL}$ Brefeldin A was added to the cells and supernatants were collected after $2 \mathrm{~h}$ for analysis of luciferase activity. D, 293 T cells were transfected with GLUC, GFP-spGLUC and $\beta$-actin-spGLUC and analyzed for luciferase activity in supernatants after 24 h. E, 293 T cells were transfected with GFP-spGLUC and BActin-spGLUC, treated with Brefeldin A and supernatants were collected and analyzed for luciferase activity as described. B-D, Shown is the mean of three independent experiments with standard error bars. R.L.U. - relative light units, SN - supernatant.

readily detectable when supernatants were mixed with PBS, and were absent following treatment with $1 \%$ SDS (Figure 3A,B). Incubation of supernatants at $95^{\circ} \mathrm{C}$ for 5 min did not result in a significant reduction of GLUC activity (Figure 3A,B). In order to test whether GLUC and dNGLUC are stable at elevated temperatures, thus facilitating the use in time kinetic measurements at physiological temperatures, the half-life of GLUC and dNGLUC at $37^{\circ} \mathrm{C}$ was determined in a timecourse experiment (Figure 3C,D). GLUC and dNGLUC activity did not show any loss of activity even after incubation for 5 days at $37^{\circ} \mathrm{C}$. Thus, GLUC and dNGLUC are very stable reporter proteins that can be used for kinetic measurements of protein expression and secretion at physiological temperatures.
Non-conventional GLUC release is not stress-induced and does not require autophagy

Recently, it has been observed that autophagy can contribute to secretion of cytoplasmic proteins by a non-conventional secretion route [28-31]. In order to determine whether autophagy is involved in the release of dNGLUC, $293 \mathrm{~T}$ cells were treated with various agents that are known to induce or inhibit autophagy (Table 1, Figure 4A), as well as Brefeldin $\mathrm{A}$ and digitoxin as autophagy independent controls. Inducers of autophagy such as rapamycin did not increase secretion of dNGLUC. Blocking autophagolysosomal fusion by treatment with chloroquine had only a minor effect on release of dNGLUC from cells (Figure 4A). None of the treatments except digitoxin had a significant effect on overall cell viability (Table 1 ), thus supporting the 

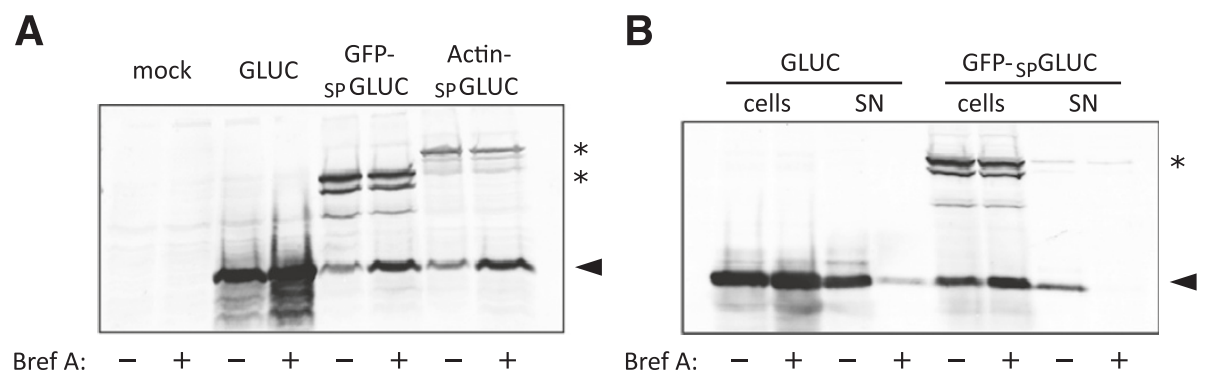

Figure 2 Bicistronic expression system for release of Gaussia luciferase activity. A, whole cell lysates from transfected 293 T cells described in Figure $1 \mathrm{E}$ were subjected to $10 \%$ PAGE and subsequent immunoblotting using a rabbit anti-GLUC antibody. Bands corresponding to GLUC are indicated by an arrowhead and bands corresponding to intact GFP-spGLUC and $\beta$-actin-spGLUC are indicated by asterisks. B, 293 T cells transfected with GLUC or GFP-sp GLUC were analyzed for GLUC activity in cell lysates and supernatants by immuno-blotting with anti-GLUC antibody. Supernatants and cell lysates were collected from the same well. Supernatants were enriched as described in Methods. Cell lysates and supernatants were resolved by PAGE and bands were detected with anti-GLUC antibody. Bands corresponding to GLUC are indicated by an arrowhead and bands corresponding to intact GFP-spGLUC are indicated by an asterisk. Experiments have been performed three times and shown are representative blots. SN - supernatant.

notion that neither induction nor inhibition of autophagosomal processes has an effect on dNGLUC release from cells.

In order to test whether cells defective in autophagy would exhibit dNGLUC release, murine embryonic fibroblasts from an inducible ATG5 knockout mouse [39] were transduced with dNGLUC and treated with doxycycline for up to 3 days. ATG5 is essential for LC3 lipidation and the formation of the autophagic membrane. After 3 days of treatment, autophagy was defective in these cells as indicated by an absence of the lipidated form of LC3, LC3-II (Figure 4B) and an absence of ATG5 (Figure 4C). Release of dNGLUC from autophagydefective cells was not decreased (Figure 4D), indicating that autophagy is not required for the release of dNGLUC.

\section{Release of Gaussia luciferase can be enhanced by stimulation with growth factors}

In order to assess whether Gaussia luciferase can be used for kinetic measurements of rapid cellular processes, the kinetics of dNGLUC release from cells were determined. The amount of dNGLUC in cell supernatants at various time points after a PBS wash and media change was measured. dNGLUC is a very sensitive reporter gene and release into supernatants was detected as early as $5 \mathrm{~min}$ after media change (Figure 5A). The amount of luciferase activity in supernatants increased in a linear manner over time up to $2 \mathrm{~h}$, indicating a steady-state release into supernatants. No sign of cell death or damage was observed, even at later time points, that might explain release. In contrast, treatment with cytotoxic agents typically reduced the amount of dNGLUC detected in supernatants, suggesting that dNGLUC release requires cell vitality (see Figure 4A).
In order to test whether release of dNGLUC from cells could be controlled by growth-promoting factors, luciferase release from cells expressing dNGLUC in serumfree media were compared to cells in complete media. A significant reduction in dNGLUC release was observed compared to that of cells cultured in complete media (Figure 5B). Next, the kinetics of release of dNGLUC in serum-free and serum-containing media was explored. As can be seen in Figure 5C, serum strongly enhanced release of dNGLUC within $1 \mathrm{~h}$ of addition to the culture, indicating an early response to growth factors. To determine whether this effect could be attributable to growth factors as opposed to other serum constituents, COS-7 cells expressing dNGLUC were treated with $100 \mathrm{ng} / \mathrm{ml}$ EGF for $2 \mathrm{~h}$ and release of dNGLUC into supernatants was analyzed. Epidermal Growth Factor alone strongly stimulated release of dNGLUC into supernatants (Figure 5D), indicating that release of dNGLUC is responsive to growth factor treatment.

\section{Non-conventional GLUC release is suppressed by GGA1}

In order to identify proteins that regulate the nonconventional secretion of GLUC, shRNA-mediated knockdown of candidate proteins involved in Golgi trafficking was examined. shRNA-mediated knockdown of a Golgiassociated protein, Golgin-Gamma-ear-Adaptin (GGA) 1 (Figure 6A) was found to significantly enhance release of dNGLUC (Figure 6B). Furthermore, overexpression of GGA1 resulted in a strong reduction of dNGLUC release (Figure 6C). As a control, the release of another unconventionally secreted protein, Fibroblast Growth Factor (FGF)-2, was investigated. Release of FGF2 from $293 \mathrm{~T}$ cells was not affected by shRNA-mediated knockdown of GGA1 (Figure 6D), in accordance with the notion that FGF2 secretion is insensitive to treatment with Brefeldin $\mathrm{A}$ and requires a plasma membrane transporter [40]. 

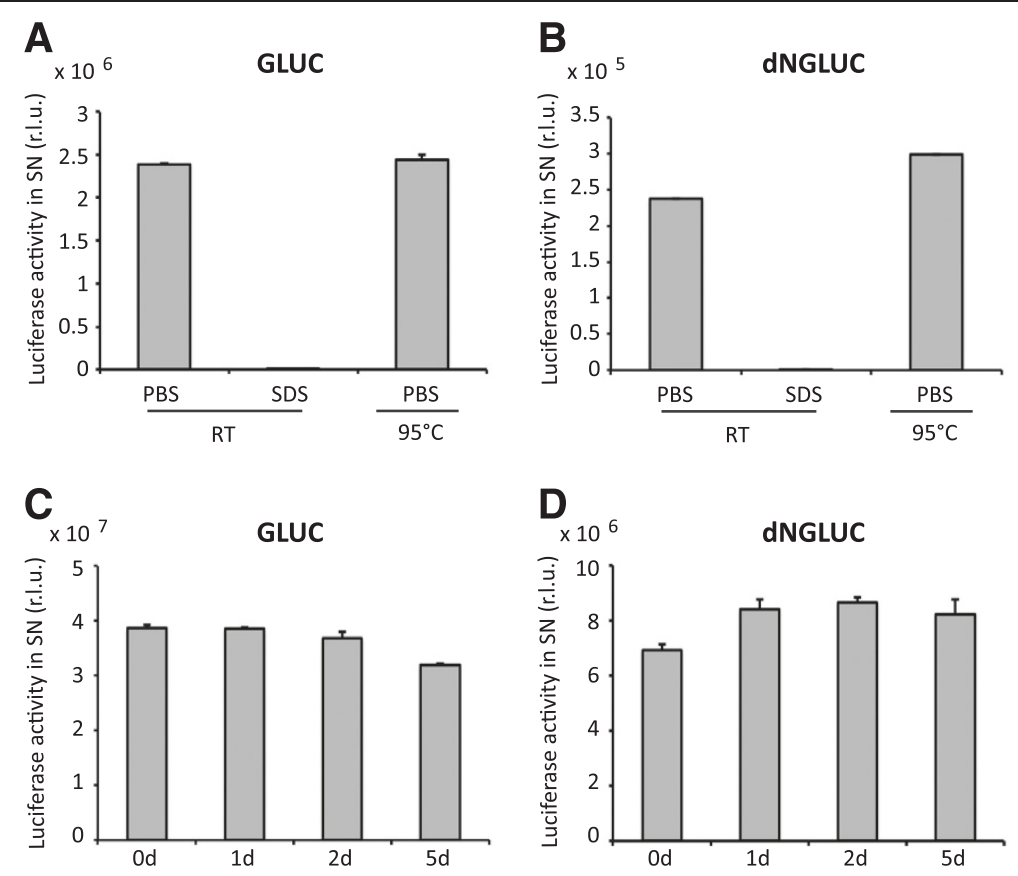

Figure 3 GLUC and dNGLUC activities are heat-stable. A, B, 293 T cells were transfected with GLUC or dNGLUC and supernatants were collected after $24 \mathrm{~h}$. $10 \mu \mathrm{L}$ of supernatant were mixed in $90 \mu \mathrm{L}$ of PBS, or PBS/1\% SDS at room temperature or in $90 \mu \mathrm{I}$ PBS incubated at $95^{\circ} \mathrm{C}$ for 5 min before analysis. C, D Supernatants from cells expressing GLUC or dNGLUC were incubated at $37^{\circ} \mathrm{C}$ for the indicated time points prior to analysis in a plate reader. A-D, shown is the mean plus standard deviations of three experiments.

R.L.U. - relative light units, SN - supernatant.

Enhanced release of dNGLUC upon shRNA-mediated knockdown of GGA1 could be reversed by transfection of a Flag-tagged GGA1 construct refractory to knockdown (GGA1m; Figure 6E). We therefore conclude that GGA1 is an endogenous inhibitor of dNGLUC release.

\section{Discussion}

Gaussia luciferase activity can be released from cells by three distinct mechanisms: 1) classical N-terminal signal peptide mediated secretion (GLUC); 2) internal signal peptide mediated release(GFP-spGLUC and actin-spGLUC);

Table 1 Effect of autophagy modulators on dNGLUC secretion

\begin{tabular}{|c|c|c|c|}
\hline Autophagy regulator & Mode of action & $\begin{array}{l}\text { dNGLUC activity in sn } \\
\text { in r.l.u }\left(\times 10^{5}\right)\end{array}$ & $\begin{array}{l}\text { Viability } \\
\text { (norm. to DMSO) }\end{array}$ \\
\hline \multicolumn{4}{|l|}{ Controls } \\
\hline DMSO & & $57.8 \pm 4.1$ & 1.0 \\
\hline No treatment & & $63.9 \pm 1.1$ & 1.0 \\
\hline Brefeldin A & Inhibition of Golgi trafficking [32] & $9.1 \pm 0.9$ & 1.0 \\
\hline Digitoxin & Inhibition of sodium-potassium ATPase [33] & $8.4 \pm 0.9$ & 0.4 \\
\hline \multicolumn{4}{|l|}{ Autophagy inhibitors } \\
\hline Chloroquine & $\begin{array}{l}\text { Prevention of lysosome acidification and lysosome } \\
\text { autophagosome fusion [34] }\end{array}$ & $44.8 \pm 5.5$ & 0.7 \\
\hline \multicolumn{4}{|l|}{ Autophagy activators } \\
\hline Trifluoperazine & Dopamine antagonist [34] & $58.1 \pm 3.1$ & 1.2 \\
\hline Rapamycin & Inhibition of mTOR [35] & $51.8 \pm 5.1$ & 0.8 \\
\hline Tamoxifen & Inhibition of mTOR signaling via increased ceramide levels $[36,37]$ & $38.1 \pm 3.6$ & 0.8 \\
\hline Thenoyltrifluoro- actetone & Inhibition of mitochondrial electron transport [38] & $61.1 \pm 3.3$ & 0.9 \\
\hline
\end{tabular}


A

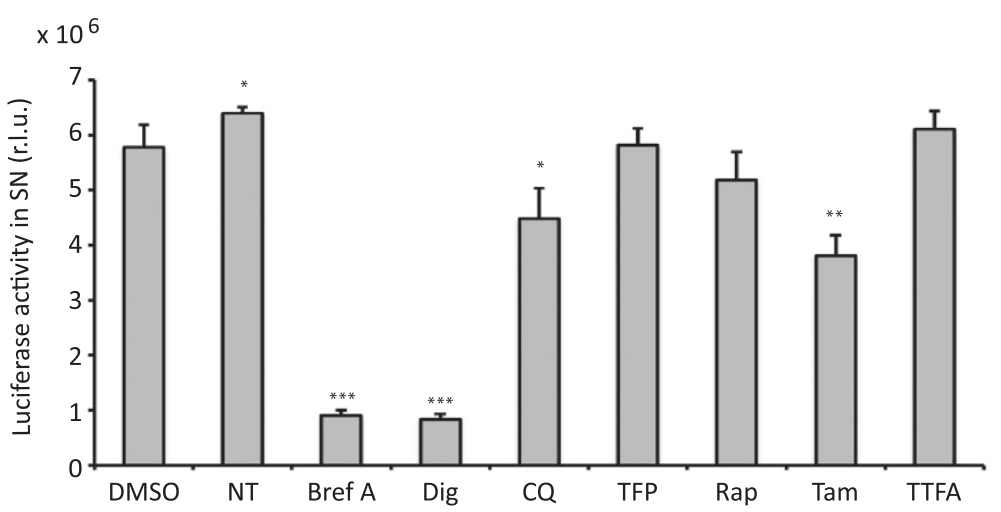

B

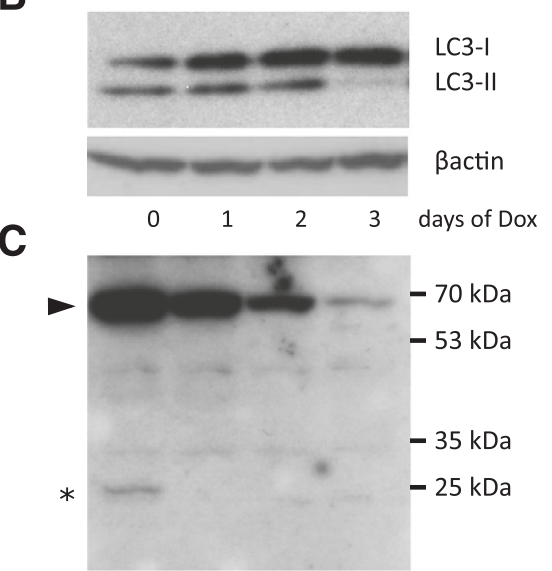

D

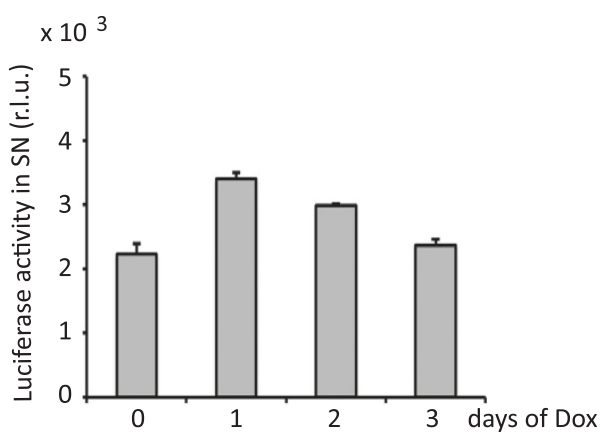

Figure 4 Release of Gaussia luciferase does not require autophagy. A, 293 T cells were treated with $10 \mu \mathrm{M}$ of digitoxin (Dig), chloroquine (CQ), Trifluorperazine (TFP), rapamycin (Rap), tamoxifen (Tam), or theonyltrifluoracetone (TTFA), $10 \mu \mathrm{g} / \mathrm{mL}$ Brefeldin A, DMSO or left untreated (NT) and analyzed for luciferase activity in supernatants ( ${ }^{*} P<0.05$; ${ }^{* *} P<0.01$; $\left.{ }^{* *} P<0.001\right)$. B, embryonic fibroblasts from ATG5 inducible knockout mice were transduced with dNGLUC. ATG5 knockout was induced using $1 \mu \mathrm{g} / \mathrm{mL}$ of doxycycline. Cells and supernatants were collected on three consecutive days after induction. Cells were washed and incubated in fresh medium for an additional $2 \mathrm{~h}$ before collection of supernatant. The efficiency of ATG5 knockout was verified by immunoblotting with detetction of LC3 (B), $\beta$-actin, and ATG5 (C). D, Luciferase activity in the correspondent supernatants was measured using the Perkin Elmer Envision II at the indicated time points. A, B, shown is the mean plus standard deviation of three experiments. Significances were calculated with a two-sided paired t-test. R.L.U. - relative light units, SN - supernatant.

and 3) non-conventional release in the absence of a signal peptide (dNGLUC). While assays based on conventional secretion of Gaussia luciferase have been previously described [41], the data presented here support two novel applications of Gaussia luciferase: (1) with the use of an internal signal peptide to monitor bicistronic gene expression and secretion and (2) use as a reporter to study non-conventional secretion mechanisms.

One advantage of secreted luciferases is their potential for deployment in multiplexed reporter assays. However, one limitation for multiplexing is the availability of expression cassettes for multiple reporter genes. To circumvent this problem, bicistronic expression systems are available such as those based on internal ribosomal entry sites (IRES) or self-cleaving peptides (e.g. the picornavirus 2A sequence [42]). However, IRES-based systems can result in inefficient expression of the downstream gene, and P2A self-cleaving peptides leave both a C-terminal extension on the upstream gene product, and an $\mathrm{N}$-terminal proline on the downstream product. It has been shown that in the presence of an $\mathrm{N}$-terminal signal peptide at the front of two sequential proteins, an internal signal sequence can function to export the two proteins from the cell [27]. The internal signal sequence can function either as a transfer signal resulting in export of the downstream peptide or alternatively as a stop-transfer signal resulting in membrane anchoring of the full peptide sequence. The bicistronic expression system proposed here based on GFP fused to Gaussia luciferase results in efficient export of the peptide downstream of the signal peptide. Interestingly, the Gaussia luciferase internal signal sequence functions in the absence of an N-terminal signal sequence as demonstrated for GFP-sp GLUC and $\beta$-actin-sp GLUC. A systematic comparison of signal sequence strength has previously shown 


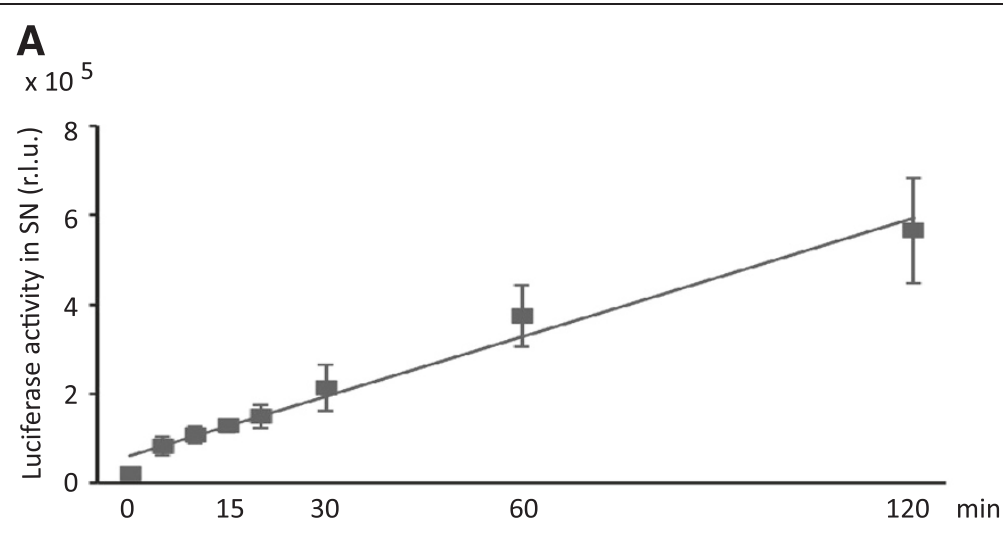

B

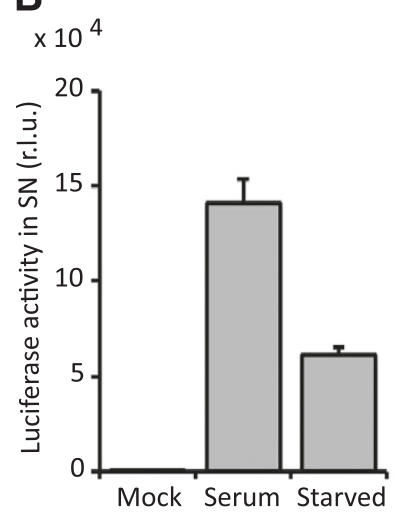

D

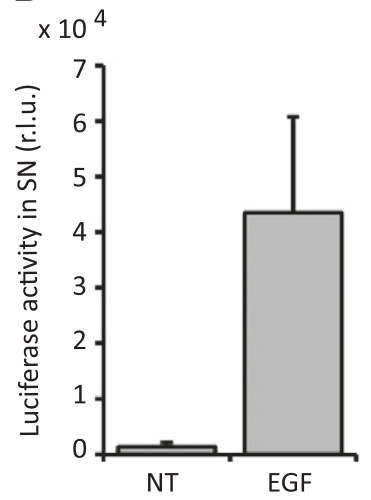

C $\times 10^{4}$

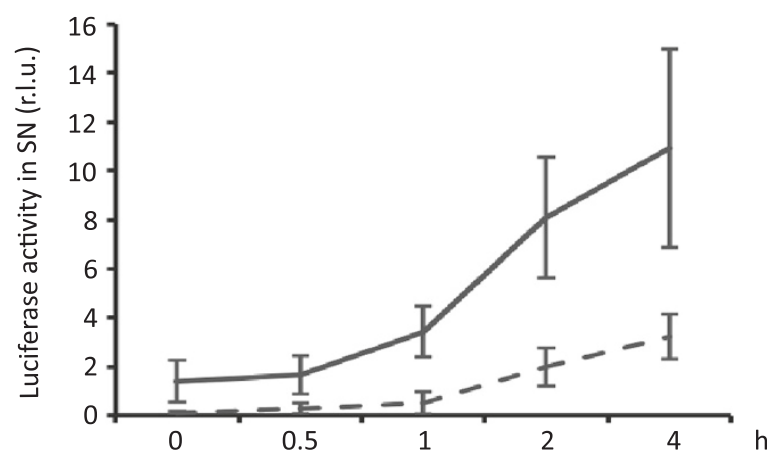

Figure 5 Secretion of dNGLUC is induced by growth factor stimulation. A, 293 T cells were transfected with dNGLUC and cultured overnight. Cells were washed once in PBS and fresh media was added. Supernatants were collected at the indicated time points prior to analysis on a PerkinElmer Envision multilabel plate reader. B, C, 293 T cells expressing dNGLUC were cultured for $24 \mathrm{~h}$ and then starved in $0.1 \%$ FCS overnight. Media containing serum (full line) or no serum (dotted line) was added back to the cells and luciferase activity in supernatants was measured after $4 \mathrm{~h}$ (B) or at the indicated time points (C) using a PerkinElmer Envision II plate reader. Shown is the mean of 5 independent transfections with standard deviation. D, COS-7 cells were transiently transfected with pEAK12-dNGLUC using calcium phosphate precipitation, cultured for $24 \mathrm{~h}$ and then starved overnight in $0.1 \%$ FCS. Cells were then stimulated with $100 \mathrm{ng} / \mathrm{mL}$ EGF at $37^{\circ} \mathrm{C}$ for $2 \mathrm{~h}$ and supernatants were harvested. C-D, shown is the mean of three experiments plus standard deviations. R.L.U. - relative light units, SN - supernatant.

that the Gaussia luciferase signal peptide is one of the most efficient secretory signals known [43], which may explain the effective export of proteins even when the signal sequence is placed between two reporter proteins. Our results indicate GLUC should have utility for bicistronic reporter gene expression and secretion.

Protein secretion is a complex process that involves intra-cellular trafficking steps and post-translational 

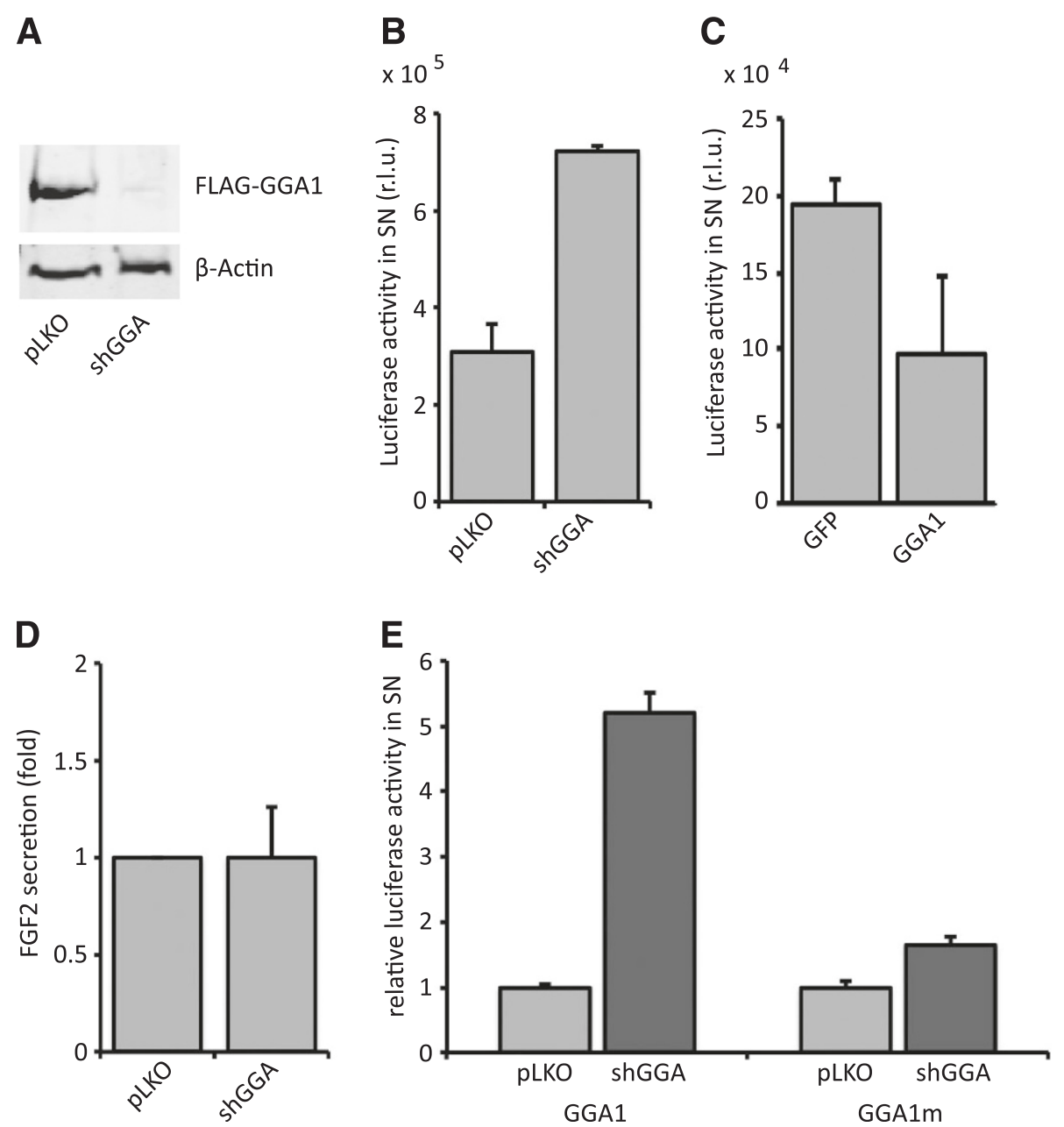

Figure 6 Release of Gaussia luciferase is suppressed by GGA1. A, To test efficiency of shRNA mediated GGA knockdown 293 T cells expressing Flag-tagged GGA1 were co-transfected with pLKO control vector or pLKO shGGA. Cell lysates were resolved on 10\% PAGE and transferred to PVDF membranes. The blots were probed with anti-Flag M2 or anti- $\beta$-actin antibody. B, 293 T cells were co-transfected with dNGLUC and the shRNA vectors pLKO (control) or pLKO shGGA and cultured for $72 \mathrm{~h}$. Supernatants were harvested and analyzed for Gaussia luciferase activity using a PerkinElmer TopCount. C, 293 T cells were co-transfected with dNGLUC and an expression vector for GFP or GGA1 and cultured for $24 \mathrm{~h}$ prior to analysis of GLUC activity in supernatants as in A. D, FGF2 was transfected into 293 T cells together with a control vector (pLKO) or an shRNA vector targeting GGA1. FGF2 secretion into supernatants was measured using an ELISA kit (R\&D Systems) after $24 \mathrm{~h}$. E, 293 T cells expressing dNGLUC were co-transfected with pLKO vector control or shGGA and wild-type Flag-GGA1 or a mutant version of GGA1 that is refractory to shRNA mediated knockdown by shGGA (GGA1m). Supernatants were harvested after $48 \mathrm{~h}$ and analysed for GLUC activity as previously described. B-E, shown are the results from three independent experiments plus standard deviation. R.L.U. - relative light units, SN - supernatant.

modifications to the secretory cargo. Canonical secretion involves translocation of the nascent polypeptide from the ribosome to the lumenal endoplasmatic reticulum (ER) followed by vesicular transport through the Golgi. This type of secretion is initiated by interaction of a hydrophobic $\mathrm{N}$-terminal signal sequence on the polypeptide with the signal recognition particle. Some proteins, however, lack an N-terminal signal peptide and are secreted by a non-conventional secretion that is insensitive to treatment with inhibitors of ER/Golgi trafficking such as Brefeldin A $[44,45]$. Proteins known to undergo non-conventional secretion include interleukin (IL) $-1 \alpha$ and $-1 \beta$, fibroblast growth factor (FGF) 1 and 2, HIV tat, transglutaminases and galectins, among others. In some cases, including those of HIV tat, FGF-2 and cellular transglutaminase, the export is constitutive, whereas in other cases secretion is initiated by a regulated event, such as the cleavage of interleukins $1 \alpha$ and $1 \beta$ by caspase- 1 . Multiple mechanisms for non-conventional secretion have been identified, including lysosomal secretion, plasma membrane shedding, exosomal release, as well as secretion through plasma membrane resident transporters [46]. In general, non-conventional secretion is poorly understood and should benefit from the application of novel tools that permit the use of unbiased approaches such as high-throughput screening technologies. We present here 
evidence that dNGLUC can be used as reporter gene to study non-conventional secretion.

Recently, an involvement of autophagy in nonconventional secretion has been described in yeast, Drosophila, mammals and plants [28-31,47]. We have shown that cellular stress responses and autophagy are not involved in dNGLUC release, because the same amount of dNGLUC was released from ATG5-/- null cells as was released from control cells. In addition, treatment with autophagy inducers or inhibitors had no effect on release of dNGLUC. The underlying mechanisms of this type of secretion are worthy of further investigation, firstly because the process is unusual and may expose new aspects of general cell biology, and secondly because such an understanding would enhance the development of reporter systems based on this type of secretion.

In this study we have also shown that GGA1 suppresses release of dNGLUC from cells. GGAs are a family of monomeric clathrin adaptors that mediate the sorting of transmembrane cargo from the trans-Golgi network to late endosomes [48]. GGA1 can directly interact with clathrin [49-51], AP1 [52,53], ARF [54], Rabaptin-5 [55,56] and with a variety of cargo molecules [57], and is required for sorting of the lysosomal targeting receptor, mannose6-phosphate receptor [48,52]. It has also been observed that overexpression of GGA3 inhibits retrovirus assembly [58], and that the molecule interacts with TSG101, an ubiquitin-binding component of the multivesicular body that is a key regulator of HIV budding. In addition, GGAs have been implicated in trafficking and processing of APP [59-62] have been found to bind to $\beta$-secretase $[44,63,64]$ and appear to restrain $A \beta$ secretion, as indicated by the enhanced secretion observed following siRNA mediated knockdown of GGA1. Here, we have observed suppression of secretion of dNGLUC by GGA1.

In principle GGA1 might suppress release of dNGLUC by interfering with lysosomal sorting. In this scenario dNGLUC might be sorted in large measure to the lysosome and consequently, suppression of lysosomal targeting would result in a net increase of flux through other trafficking routes. However, lysosomal inhibitors such as chloroquine had no effect on release of dNGLUC, diminishing the attractiveness of this hypothesis.

\section{Conclusions}

In summary, we present here a novel type of secretion that occurs in the absence of a signal peptide, can ferry diverse cargo to the extracellular space, is enhanced by growth factor stimulation and does not require autophagy. In the future, it will be desirable to address the molecular mechanisms of this type of secretion in more detail. The secreted luciferase presented here should be an excellent tool to expose additional features of the mechanism using high-throughput screening technologies.

\section{Methods}

\section{Plasmids and constructs}

pEAK12-dNGLUC, GFP-dNGLUC and Actin-LC3-dNGLUC have been described previously. GFP-spGLUC and Actin-spGLUC were generated by inserting the wild-type GLUC sequence into pEAK12-GFP and pEAK12-ActinLC3-dNGLUC between EcoRI and Not $I$ restriction sites. GGA1 was amplified by PCR using primers 5'-GACG AATTCATGGAGCCCGCGATGG-3' and 5'- GACGCGG CCGCCTAGAGGCTACCCCAGG-3' and inserted via EcoRI/Not I into pEAK13-Flag. A GGA1 mutant that is refractory to siRNA treatment was generated by overlap extension PCR using the following internal primers: cctccaAgAccTaaAaatgtgA and ccacatttttaggtcttggagg. pLKO shRNA vectors were obtained from SigmaAldrich. The sequence for knockdown of GGA1 was: CGGCCG AAGAATGTGATCTTTGAA.

\section{Cell culture}

293 T and COS-7 cells were cultured in Dulbecco's modified essential media (DMEM, Gibco) supplemented with $10 \%$ fetal bovine serum (Sigma-Aldrich), 1\% GlutaMAX (Gibco) and 1\% penicillin/streptomycin (Gibco). Doxycyclineinducible ATG5-/- murine embryonic fibroblasts (MEF) [39] were obtained from Dr. Noboru Mizushima (Tokyo Dental Institute) and were cultured in DMEM (Gibco) supplemented with $1 \%$ GlutaMAX, $1 \%$ penicillin/streptomycin, $1 \%$ sodium pyruvate (PAA) and 10\% FBS (Sigma-Aldrich). Cells were induced with $1 \mu \mathrm{g} / \mathrm{ml}$ doxycycline (SigmaAldrich) for the indicated time points.

\section{Small molecule compound treatment}

All compounds were obtained from Sigma-Aldrich and dissolved in DMSO as $10 \mathrm{mM}$ stock solutions unless otherwise indicated. Brefeldin A was purchased as a solution $(10 \mathrm{mg} / \mathrm{ml}$ ) from Sigma-Aldrich. Chloroquine (SigmaAldrich) was kept as a $25 \mathrm{mM}$ stock solution in water. 293 T cells expressing dNGLUC were washed once with culture medium before addition of compounds in complete DMEM. Concentrations and time of incubation are detailed in the Figure legends. Epidermal Growth Factor (EGF) was obtained from PeproTech. COS-7 cells were starved overnight in $0.1 \%$ serum and stimulated with $100 \mathrm{ng} / \mathrm{ml}$ EGF at $37^{\circ} \mathrm{C}$.

\section{Transfection and retroviral transduction}

$293 \mathrm{~T}$ cells were transfected with expression plasmids using calcium phosphate precipitation as described [65] or Lipofectamine 2000 (Invitrogen) at 200 ng/well in a 96well plate. DNA was mixed with $0.5 \mathrm{ml}$ Lipofectamine in 
$50 \mathrm{~mL}$ serum-free DMEM and incubated for $30 \mathrm{~min}$ at room temperature before addition to cells.

To express dNGLUC in MEFs, pMOWSdSV-dNGLUC was used for retroviral transduction. Retroviral stocks were prepared by transient transfection of 293 T cells. A solution consisting of $1 \mathrm{~mL}$ DMEM, $75 \mu \mathrm{L}$ of $1 \mathrm{mg} / \mathrm{mL}$ polyethylenimine, $5 \mu \mathrm{g}$ of each of the viral helper plasmids VSV-G and Gag Pol and $20 \mu \mathrm{g}$ of pMOWSdSV-dNGLUC was prepared, incubated for 20 minutes and added to a $10 \mathrm{~cm}$ plate of 293 T cells at $60 \%$ confluency. The following day the culture medium was replaced with $7 \mathrm{~mL}$ of fresh culture medium. The resulting virus-containing supernatant was collected two days after transfection and filtered through a $0.45 \mu \mathrm{m}$ cellulose acetate filter. Upon addition of polybrene to a final concentration of $1 \mu \mathrm{g} / \mathrm{mL}$, the virus solution was added to a $6 \mathrm{~cm}$ plate of MEFs at $70 \%$ confluency. After $24 \mathrm{~h}$ the solution was exchanged with regular culture media.

\section{Protein concentration}

293 T cells were cultured in serum-free medium overnight, supernatants were collected, filtered through $0.45 \mu \mathrm{m}$ filter and $500 \mu \mathrm{l}$ portions were loaded onto Amicon Ultra spin prep columns (Millipore) with a cut-off of $3000 \mathrm{Da}$. Samples were spun at $14,000 \mathrm{rpm}$ and $4^{\circ} \mathrm{C}$ for $60 \mathrm{~min}$ prior to elution of concentrated protein samples. Samples were then mixed with sample buffer and analyzed by immuno-blotting. The volume was concentrated $\sim 10$-fold.

\section{Luciferase assay}

Native Coelenterazine (Biotium) was dissolved at a concentration of $1 \mathrm{mg} / \mathrm{mL}$ in acidified Methanol. An assay buffer was prepared by mixing the coelenterazine solution 1:100 with Gaussia luciferase buffer (0.1\% disodium phosphate, $5 \%$ glycerol, $150 \mathrm{mM}$ sodium bromide, $1 \mathrm{mM}$ EDTA, $25 \mathrm{mM}$ Tris- $\mathrm{HCl} \mathrm{pH} 8$ and $2 \mathrm{mM}$ ascorbic acid). $10 \mu \mathrm{L}$ of supernatants were transferred to black 96-well plates and mixed with $90 \mu \mathrm{L}$ assay buffer prior to analysis of luciferase activity in a PerkinElmer Envision II plate photometer.

\section{Cell viability assay}

An assay solution was prepared by diluting Cell Counting Kit-8 stock solution (Sigma) 1:50 in PBS. Culture medium was aspirated from $293 \mathrm{~T}$ cells in 96 well plates and replaced with $100 \mu \mathrm{L}$ of the assay solution. After $2 \mathrm{~h}$ incubation at $37^{\circ} \mathrm{C}$ the absorbance at $450 \mathrm{~nm}$ was measured with a PerkinElmer Envision II.

\section{Elisa}

Fibroblast Growth Factor 2 (FGF2) in supernatants was measured with a R\&D Systems ELISA kit according to the manufacturer's instructions.

\section{Immuno-blotting}

$293 \mathrm{~T}$ cells were lysed in NP40 lysis buffer (50 mM Tris- $\mathrm{HCl} \mathrm{pH} 8,0.5 \%$ NP40, 0.1\% EDTA, 10\% glycerol and protease inhibitors), mixed with $2 \times$ SDS sample buffer and loaded onto 10\% PAGE pre-cast gels (BioRad). Proteins were transferred to a PVDF membrane and subsequently blocked for 1 to $2 \mathrm{~h}$ in blocking solution (5\% skimmed milk and $0.1 \%$ Tween in PBS). Proteins of interest were probed with primary antibodies overnight at $4^{\circ} \mathrm{C}$ in blocking solution. HRP-conjugated secondary antibody incubation was conducted for $1 \mathrm{~h}$ at room temperature in blocking solution. Antibody bound proteins were detected using ECL reagents. The following antibodies were used: anti-GLUC (Ketteler et al. [19,20], anti-Flag M2 (Sigma-Aldrich), anti- $\beta$-Actin (Abcam), anti-LC3 (Sigma-Aldrich), anti-ATG5 (Nanotools), HRP anti-mouse (Santa-Cruz) and HRP anti-rabbit (Santa-Cruz).

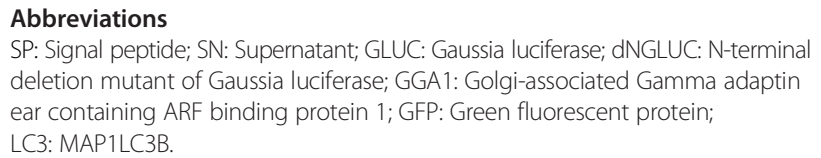

\section{Competing interests}

The authors declare that they have no competing interests.

\section{Authors' contributions}

$\mathrm{CL}$ performed experiments and helped to draft the manuscript. JF performed and designed experiments. DE, NAT, JH and IL performed experiments. JKV helped with statistical analysis and design of experiments. BS and RK conceived the study and designed experiments. RK performed experiments and wrote the manuscript. All authors read and approved the final manuscript.

\section{Acknowledgements}

JKV was supported by the Marie Curie Reintegration grant. We thank Noboru Mizushima for providing inducible ATG5 knockout MEFs. This work was supported by the Medical Research Council and BBSRC (BB/J015881/1).

\section{Author details}

${ }^{1}$ Medical Research Council, Laboratory for Moleclar and Cell Biology, University College London, Gower Street, London WC1E 6BT, UK.

${ }^{2}$ Massachusetts General Hospital, Center for Computational and Integrative Biology, Boston MA02114, USA.

Received: 25 February 2014 Accepted: 5 July 2014 Published: 9 July 2014

\section{References}

1. Thompson EM, Nagata S, Tsuji Fl: Cloning and expression of cDNA for the luciferase from the marine ostracod Vargula hilgendorfii. Proc Natl Acad Sci U S A 1989, 86(17):6567-6571.

2. Verhaegent M, Christopoulos TK: Recombinant Gaussia luciferase. Overexpression, purification, and analytical application of a bioluminescent reporter for DNA hybridization. Anal Chem 2002, 74(17):4378-4385.

3. Markova SV, Golz S, Frank LA, Kalthof B, Vysotski ES: Cloning and expression of cDNA for a luciferase from the marine copepod Metridia longa. A novel secreted bioluminescent reporter enzyme. J Biol Chem 2004, 279(5):3212-3217.

4. Takenaka Y, Masuda H, Yamaguchi A, Nishikawa S, Shigeri Y, Yoshida Y, Mizuno $H$ : Two forms of secreted and thermostable luciferases from the marine copepod crustacean, Metridia pacifica. Gene 2008, 425(1-2):28-35. 
5. Takenaka Y, Yamaguchi A, Tsuruoka N, Torimura M, Gojobori T, Shigeri Y: Evolution of bioluminescence in marine planktonic copepods. Mol Biol Evol 2012, 29(6):1669-1681.

6. Takenaka Y, Noda-Ogura A, Imanishi T, Yamaguchi A, Gojobori T, Shigeri Y: Computational analysis and functional expression of ancestral copepod luciferase. Gene 2013, 528(2). Epub 2013 Jul 2023.

7. Hall MP, Unch J, Binkowski BF, Valley MP, Butler BL, Wood MG, Otto P, Zimmerman K, Vidugiris $G$, Machleidt T, Robers MB, Benink HA, Eggers $C T$, Slater MR, Meisenheimer PL, Klaubert DH, Fan F, Encell LP, Wood KV: Engineered luciferase reporter from a deep sea shrimp utilizing a novel imidazopyrazinone substrate. ACS Chem Biol 2012, 7(11):1848-1857.

8. Maguire CA, Deliolanis NC, Pike L, Niers JM, Tjon-Kon-Fat L-A, Sena-Esteves M, Tannous BA: Gaussia luciferase variant for high-throughput functional screening applications. Anal Chem 2009, 81(16):7102-7106.

9. Degeling MH, Bovenberg MSS, Lewandrowski GK, de Gooijer MC, Vleggeert-Lankamp CLA, Tannous M, Maguire CA, Tannous BA: Directed molecular evolution reveals Gaussia luciferase variants with enhanced light output stability. Anal Chem 2013, 85(5):3006-3012.

10. Welsh JP, Patel KG, Manthiram K, Swartz JR: Multiply mutated Gaussia luciferases provide prolonged and intense bioluminescence. Biochem Biophys Res Commun 2009, 389(4):563-568.

11. Tannous BA: Gaussia luciferase reporter assay for monitoring biological processes in culture and in vivo. Nat Protoc 2009, 4(4):582-591.

12. Kim SB, Sato M, Tao H: Split Gaussia luciferase-based bioluminescence template for tracing protein dynamics in living cells. Anal Chem 2009, 81(1):67-74

13. Remy I, Michnick SW: A highly sensitive protein-protein interaction assay based on Gaussia luciferase. Nat Methods 2006, 3(12):977-979.

14. Luker KE, Mihalko LA, Schmidt BT, Lewin SA, Ray P, Shcherbo D, Chudakov DM, Luker GD: In vivo imaging of ligand receptor binding with Gaussia luciferase complementation. Nat Med 2011, 18(1):172-177.

15. Neveu G, Cassonnet P, Vidalain P-O, Rolloy C, Mendoza J, Jones L, Tangy F, Muller M, Demeret C, Tafforeau L, Lotteau V, Rabourdin-Combe C, Travé G, Dricot A, Hill DE, Vidal M, Favre M, Jacob Y: Comparative analysis of virus-host interactomes with a mammalian high-throughput protein complementation assay based on Gaussia princeps luciferase. Methods 2012, 58(4):349-359.

16. Hashimoto T, Adams KW, Fan Z, McLean PJ, Hyman BT: Characterization of oligomer formation of amyloid-beta peptide using a split-luciferase complementation assay. J Biol Chem 2011, 286(31):27081-27091.

17. Wurdinger T, Badr C, Pike L, de Kleine R, Weissleder R, Breakefield XO, Tannous BA: A secreted luciferase for ex vivo monitoring of in vivo processes. Nat Methods 2008, 5(2):171-173.

18. Bartok E, Bauernfeind F, Khaminets MG, Jakobs C, Monks B, Fitzgerald KA, Latz E, Hornung V: iGLuc: a luciferase-based inflammasome and protease activity reporter. Nat Methods 2013, 10(2):147-154

19. Ketteler R, Sun Z, Kovacs KF, He W-W, Seed B: A pathway sensor for genome-wide screens of intracellular proteolytic cleavage. Genome Biol 2008, 9(4):R64.

20. Ketteler R, Seed B: Quantitation of autophagy by luciferase release assay Autophagy 2008, 4(6):801-806.

21. Niers JM, Kerami M, Pike L, Lewandrowski G, Tannous BA: Multimodal in vivo imaging and blood monitoring of intrinsic and extrinsic apoptosis. Mol Ther 2011, 19(6):1090-1096.

22. Koutsoudakis G, Perez-del-Pulgar S, Gonzalez P, Crespo G, Navasa M, Forns X: A Gaussia luciferase cell-based system to assess the infection of cell culture- and serum-derived hepatitis C virus. PLoS One 2012, 7(12):e53254

23. Kuang E, Okumura CYM, Sheffy-Levin S, Varsano T, Shu VC-W, Qi J, Niesman IR, Yang H-J, Lopez-Otin C, Yang WY, Reed JC, Broday L, Nizet V, Ronai ZA: Regulation of ATG4B stability by RNF5 limits basal levels of autophagy and influences susceptibility to bacterial infection. PLoS Genet 2012, 8(10):e1003007.

24. Garcia-Garcia A, Rodriguez-Rocha H, Tseng MT, de Montes Oca-Luna R, Zhou HS, McMasters KM, Gomez-Gutierrez JG: E2F-1 lacking the transcriptional activity domain induces autophagy. Cancer Biol Ther 2012, 13(11):1091-1101.

25. Rodriguez-Rocha H, Gomez-Gutierrez JG, Garcia-Garcia A, Rao X-M, Chen L, McMasters KM, Zhou HS: Adenoviruses induce autophagy to promote virus replication and oncolysis. Virology 2011, 416(1-2):9-15.

26. Bhatelia K, Singh A, Tomar D, Singh K, Sripada L, Chagtoo M, Prajapati P, Singh R, Godbole MM, Singh R: Antiviral signaling protein MITA acts as a tumor suppressor in breast cancer by regulating NF-kappaB induced cell death. Biochim Biophys Acta 2014, 1842(2):144-153.

27. Coleman J, Inukai $M$, Inouye $M$ : Dual functions of the signal peptide in protein transfer across the membrane. Cell 1985, 43(1):351-360.

28. Bruns C, McCaffery JM, Curwin AJ, Duran JM, Malhotra V: Biogenesis of a novel compartment for autophagosome-mediated unconventional protein secretion. J Cell Biol 2011, 195(6):979-992.

29. Dupont N, Jiang S, Pilli M, Ornatowski W, Bhattacharya D, Deretic V: Autophagy-based unconventional secretory pathway for extracellular delivery of IL-1beta. EMBO J 2011, 30(23):4701-4711.

30. Duran JM, Anjard C, Stefan C, Loomis WF, Malhotra V: Unconventional secretion of Acb1 is mediated by autophagosomes. J Cell Biol 2010, 188(4):527-536.

31. Manjithaya R, Anjard C, Loomis WF, Subramani S: Unconventional secretion of Pichia pastoris Acb1 is dependent on GRASP protein, peroxisomal functions, and autophagosome formation. J Cell Biol 2010, 188(4):537-546.

32. Misumi Y, Miki K, Takatsuki A, Tamura G, Ikehara Y: Novel blockade by brefeldin A of intracellular transport of secretory proteins in cultured rat hepatocytes. J Biol Chem 1986, 261(24):11398-11403.

33. Glynn IM: The Action of Cardiac Glycosides on lon Movements. Pharmacol Rev 1964, 16:381-407.

34. Zhang L, Yu J, Pan H, Hu P, Hao Y, Cai W, Zhu H, Yu AD, Xie X, Ma D, Yuan J: Small molecule regulators of autophagy identified by an image-based high-throughput screen. Proc Natl Acad Sci U S A 2007, 104(48):19023-19028.

35. Noda T, Ohsumi Y: Tor, a phosphatidylinositol kinase homologue, controls autophagy in yeast. J Biol Chem 1998, 273(7):3963-3966.

36. Bursch W, Ellinger A, Kienzl $H$, Torok L, Pandey S, Sikorska M, Walker R, Hermann RS: Active cell death induced by the anti-estrogens tamoxifen and ICI 164384 in human mammary carcinoma cells (MCF-7) in culture: the role of autophagy. Carcinogenesis 1996, 17(8):1595-1607.

37. Scarlatti F, Bauvy C, Ventruti A, Sala G, Cluzeaud F, Vandewalle A, Ghidoni R, Codogno P: Ceramide-mediated macroautophagy involves inhibition of protein kinase B and up-regulation of beclin 1. J Biol Chem 2004, 279(18):18384-18391.

38. Chen Y, McMillan-Ward E, Kong J, Israels SJ, Gibson SB: Mitochondrial electron-transport-chain inhibitors of complexes I and II induce autophagic cell death mediated by reactive oxygen species. J Cell Sci 2007, 120(Pt 23):4155-4166.

39. Hosokawa N, Hara Y, Mizushima N: Generation of cell lines with tetracycline-regulated autophagy and a role for autophagy in controlling cell size. FEBS Lett 2006, 580(11):2623-2629.

40. Nickel W: Unconventional secretion: an extracellular trap for export of fibroblast growth factor 2. J Cell Sci 2007, 120(Pt 14):2295-2299.

41. Badr CE, Hewett JW, Breakefield XO, Tannous BA: A highly sensitive assay for monitoring the secretory pathway and ER stress. PLoS One 2007, 2(6):e571.

42. Szymczak AL, Workman CJ, Wang Y, Vignali KM, Dilioglou S, Vanin EF, Vignali DAA: Correction of multi-gene deficiency in vivo using a single 'self-cleaving' 2A peptide-based retroviral vector. Nat Biotechnol 2004, 22(5):589-594

43. Knappskog S, Ravneberg H, Gjerdrum C, Trosse C, Stern B, Pryme IF: The level of synthesis and secretion of Gaussia princeps luciferase in transfected $\mathrm{CHO}$ cells is heavily dependent on the choice of signal peptide. J Biotechnol 2007, 128(4):705-715.

44. Prudovsky I, Mandinova A, Soldi R, Bagala C, Graziani I, Landriscina M, Tarantini F, Duarte M, Bellum S, Doherty H, Maciag T: The non-classical export routes: FGF1 and IL-1alpha point the way. J Cell Sci 2003, 116(Pt 24):4871-4881.

45. Nickel W: The mystery of nonclassical protein secretion. A current view on cargo proteins and potential export routes. Eur J Biochem 2003, 270(10):2109-2119

46. Nickel W, Rabouille C: Mechanisms of regulated unconventional protein secretion. Nat Rev Mol Cell Biol 2009, 10(2):148-155

47. Pallet N, Sirois I, Bell C, Hanafi L-A, Hamelin K, Dieude M, Rondeau C, Thibault P, Desjardins M, Hebert M-J: A comprehensive characterization of membrane vesicles released by autophagic human endothelial cells. Proteomics 2013, 13(7):1108-1120.

48. Hirst J, Lui WW, Bright NA, Totty N, Seaman MN, Robinson MS: A family of proteins with gamma-adaptin and VHS domains that facilitate trafficking between the trans-Golgi network and the vacuole/lysosome. J Cell Biol 2000, 149(1):67-80 
49. Knuehl C, Chen CY, Manalo V, Hwang PK, Ota N, Brodsky FM: Novel binding sites on clathrin and adaptors regulate distinct aspects of coat assembly. Traffic 2006, 7(12):1688-1700.

50. Puertollano R, van der Wel NN, Greene LE, Eisenberg E, Peters PJ, Bonifacino JS: Morphology and dynamics of clathrin/GGA1-coated carriers budding from the trans-Golgi network. Mol Biol Cell 2003, 14(4):1545-1557.

51. Zhang F, Yim YI, Scarselletta S, Norton M, Eisenberg E, Greene LE: Clathrin adaptor GGA1 polymerizes clathrin into tubules. J Biol Chem 2007, 282(18):13282-13289.

52. Doray B, Ghosh P, Griffith J, Geuze HJ, Kornfeld S: Cooperation of GGAs and AP-1 in packaging MPRs at the trans-Golgi network. Science 2002, 297(5587):1700-1703.

53. Zhdankina O, Strand NL, Redmond JM, Boman AL: Yeast GGA proteins interact with GTP-bound Arf and facilitate transport through the Golgi. Yeast 2001, 18(1):1-18.

54. Shiba T, Kawasaki M, Takatsu H, Nogi T, Matsugaki N, Igarashi N, Suzuki M, Kato R, Nakayama K, Wakatsuki S: Molecular mechanism of membrane recruitment of GGA by ARF in lysosomal protein transport. Nat Struct Biol 2003, 10(5):386-393.

55. Mattera R, Arighi CN, Lodge R, Zerial M, Bonifacino JS: Divalent interaction of the GGAs with the Rabaptin-5-Rabex-5 complex. EMBO J 2003, 22(1):78-88.

56. Zhu G, Zhai P, He X, Wakeham N, Rodgers K, Li G, Tang J, Zhang XC: Crystal structure of human GGA1 GAT domain complexed with the GAT-binding domain of Rabaptin5. EMBO J 2004, 23(20):3909-3917.

57. Mattera R, Puertollano R, Smith WJ, Bonifacino JS: The trihelical bundle subdomain of the GGA proteins interacts with multiple partners through overlapping but distinct sites. J Biol Chem 2004, 279(30):31409-31418.

58. Joshi A, Nagashima K, Freed EO: Defects in cellular sorting and retroviral assembly induced by GGA overexpression. BMC Cell Biol 2009, 10:72.

59. von Arnim CA, Tangredi MM, Peltan ID, Lee BM, Irizarry MC, Kinoshita A, Hyman BT: Demonstration of BACE (beta-secretase) phosphorylation and its interaction with GGA1 in cells by fluorescence-lifetime imaging microscopy. J Cell Sci 2004, 117(Pt 22):5437-5445.

60. Wahle T, Prager K, Raffler N, Haass C, Famulok M, Walter J: GGA proteins regulate retrograde transport of BACE1 from endosomes to the trans-Golgi network. Mol Cell Neurosci 2005, 29(3):453-461.

61. Wahle T, Thal DR, Sastre M, Rentmeister A, Bogdanovic N, Famulok M, Heneka MT, Walter J: GGA1 is expressed in the human brain and affects the generation of amyloid beta-peptide. J Neurosci 2006, 26(49):12838-12846.

62. von Arnim CAF, Spoelgen R, Peltan ID, Deng M, Courchesne S, Koker M, Matsui T, Kowa H, Lichtenthaler SF, Irizarry MC, Hyman BT: GGA1 acts as a spatial switch altering amyloid precursor protein trafficking and processing. J Neurosci 2006, 26(39):9913-9922.

63. He X, Chang WP, Koelsch G, Tang J: Memapsin 2 (beta-secretase) cytosolic domain binds to the VHS domains of GGA1 and GGA2: implications on the endocytosis mechanism of memapsin 2. FEBS Lett 2002, 524(1-3):183-187.

64. Shiba T, Kametaka S, Kawasaki M, Shibata M, Waguri S, Uchiyama Y, Wakatsuki S: Insights into the phosphoregulation of beta-secretase sorting signal by the VHS domain of GGA1. Traffic 2004, 5(6):437-448.

65. Ketteler R, Glaser S, Sandra O, Martens UM, Klingmuller U: Enhanced transgene expression in primitive hematopoietic progenitor cells and embryonic stem cells efficiently transduced by optimized retroviral hybrid vectors. Gene Ther 2002, 9(8):477-487.

doi:10.1186/1471-2091-15-14

Cite this article as: Luft et al: Application of Gaussia luciferase in bicistronic and non-conventional secretion reporter constructs. BMC Biochemistry 2014 15:14.

\section{Submit your next manuscript to BioMed Central and take full advantage of:}

- Convenient online submission

- Thorough peer review

- No space constraints or color figure charges

- Immediate publication on acceptance

- Inclusion in PubMed, CAS, Scopus and Google Scholar

- Research which is freely available for redistribution

Submit your manuscript at www.biomedcentral.com/submit 